EXTENDED REPORT

\title{
Treated threshold stage 3 versus spontaneously regressed subthreshold stage 3 retinopathy of prematurity: a study of motility, refractive, and anatomical outcomes at 6 months and 36 months
}

\author{
J Sahni, N V Subhedar, D Clark
}

Br J Ophthalmol 2005;89:154-159. doi: 10.1136/bjo.2004.045815

\begin{abstract}
Aim: To compare the visual acuity (VA), spherical equivalent refractive error, motility, and anatomical outcomes in children with treated regressed threshold stage 3 retinopathy of prematurity (ROP) and those with spontaneously regressed subthreshold stage 3 ROP.

Method: 6 month and 3 year data collected from infants examined between 1989 and 1999 with regressed stage $3 \mathrm{ROP}$, with or without treatment were retrospectively reviewed.

Results: 85 infants were included in this study. 40 eyes received cryotherapy, 81 eyes laser photocoagulation, and 34 eyes had spontaneously regressed subthreshold stage 3 ROP. Grating acuity score $\geqslant 2$ cycles/degree (c/d) at 6 months was predictive of optotype acuity $\geqslant 6 / 9$ in $69 \%$ of eyes and a score $<2 \mathrm{c} / \mathrm{d}$ at 6 months was predictive of acuity $<6 / 9$ in $88 \%$ of eyes. Eyes with subthreshold stage 3 ROP were twice as likely to have VA of $6 / 9$ or better at 36 months than the treated eyes. The mean spherical equivalent refractive error at 36 months was -6.5 dioptres (D) $(-21.5 \mathrm{D}$ to $+1.38 \mathrm{D})$ in cryotherapy treated eyes, $-2.4 \mathrm{D}(-13 \mathrm{D}$ to $+4 \mathrm{D})$ in the laser group, and $-0.22 \mathrm{D}(-9 \mathrm{D}$ to $+2.25 \mathrm{D})$ in the subthreshold group. Eyes within the treated groups were more myopic than the eyes within the spontaneously regressed group $(p=0.005)$. At 36 months, 42 out of the 85 infants (that is, $49 \%$ ) had strabismus (44\% in the cryotherapy group, $26 \%$ in the laser group, and $25 \%$ in the subthreshold group). There was a statistically significant association between the presence of strabismus and anisometropia $(p=0.016)$ and strabismus and intraventricular haemorrhage (IVH) $(p=0.005)$. There was a statistically significant difference in the incidence of strabismus between mild and moderate and severe grade IVH $(p=0.01)$. Eight out of 40 eyes in the cryotherapy group and six out of the 81 eyes in the laser group developed macular ectopia. None of the eyes in the spontaneously regressed group had macular dragging.

Conclusions: In this study, the grating acuity at 6 months was a good predictor of the 3 year optotype acuity in all groups. Eyes with spontaneously regressed subthreshold stage 3 ROP were associated with better vision at 3 years of age and a lesser degree of myopia compared to the treated groups. Strabismus developed predominantly in the treated groups and was frequently associated with neurological damage and/or anisometropia. The spontaneously regressed subthreshold stage 3 group had a better anatomical outcome compared to the groups in which the retinopathy regressed following treatment.
\end{abstract}

Accepted for publication 22 July 2004

$\mathrm{T}$ he Cryotherapy for Retinopathy of Prematurity Cooperative Group demonstrated a beneficial effect of treating threshold stage 3 ROP. ${ }^{1}$ During the past several years, studies have demonstrated that laser photocoagulation for threshold ROP was more likely to result in a better anatomical and visual outcome and less myopia compared to cryotherapy. ${ }^{2-5}$

Recent studies $^{6-9}$ including ETROP (Early Treatment for ROP Cooperative Group) study showed improved outcome following treatment for subthreshold ROP. However, long term follow up results are not currently available for the ETROP study and few studies have reviewed the structural and functional outcome in eyes with spontaneously regressed subthreshold stage 3 ROP. Our report examines the change in vision and refractive error at 6 months post-term and 36 months post-term in 85 children and compares the vision, refractive and orthoptic outcome between the two different regressed ROP groups with and without treatment (threshold stage 3 ROP and subthreshold stage 3 ROP).

\section{PATIENTS AND METHODS}

In the period between 1989 and 1999, 1820 preterm babies, with birth weights less than $1500 \mathrm{~g}$ and or less than or equal

to 31 weeks gestational age were screened for ROP based on Royal College of Ophthalmologists and the British Association of Perinatal Medicine guidelines (1995) at the Merseyside Regional Neonatal Intensive Care Unit (a tertiary referral unit for ROP treatment) by a single clinician (DC). The first examination was between 6 and 7 weeks postnatal age. Subsequent examinations continued until vascularisation was complete or the retinopathy showed no further signs of progression.

Treatment was undertaken when threshold was reached. The threshold for treatment was defined as 5 contiguous or 8 or more cumulative clock hours of stage 3 in zone I or zone II, in the presence of "plus" disease. Subthreshold was defined as stage 3, but not meeting the above definition.

The data were collected retrospectively from a database where the data were recorded prospectively. The following clinical parameters were recorded at each examination.

Abbreviations: $c / d$, cycles/degree; IVH, intraventricular haemorrhage; $\mathrm{PVL}$, periventricular leucomalacia; ROP, retinopathy of prematurity; VA, visual acuity 


\section{Vision}

Visual acuity (VA) was recorded using Teller grating acuity cards at 6 months post-term and Kay pictures at 36 months post-term. Refractive error was corrected for if myopia exceeded $-4 \mathrm{D} .{ }^{6}$ VA was scored as $<2$ cycles/degree (c/d) or $\geqslant 2 \mathrm{c} / \mathrm{d}$ at the 6 month examination and $<6 / 9$ or $\geqslant 6 / 9$ at the 36 month examination. A VA of $\geqslant 2 \mathrm{c} / \mathrm{d}$ at 6 months and $6 / 9$ or better at 3 years was considered a favourable outcome. ${ }^{10}$

\section{Refraction}

An experienced optometrist determined the refractive errors after cycloplegic retinoscopy using $1 \%$ cyclopentolate hydrochloride at each examination and the spherical equivalent (SE) was calculated. Eyes with $5.00 \mathrm{D}$ or more of myopia were considered to have high myopia. ${ }^{11}$

\section{Orthoptic assessment}

Cover test, prism cover test, and ocular movements were assessed by a senior orthoptist at every visit. Presence or absence of strabismus and nystagmus was documented.

\section{Cranial ultrasound}

The occurrence of intraventricular haemorrhage (IVH) and periventricular leucomalacia (PVL) was documented on cranial ultrasound. IVH was graded as $0=$ normal; $\mathrm{I}=$ subependymal haemorrhage; $\mathrm{II}=\mathrm{IVH}$ only; $\mathrm{III}=$ intraventricular haemorrhage and dilatation; IV = intraparenchymal lesion. PVL was graded as $0=$ normal; $\mathrm{I}=$ persistent periventricular flare; II = small cysts; III = large, widespread cysts. Ocular outcomes of infants with no or mild to moderate (grades I-II) haemorrhages were compared with those of infants with severe grade (III and IV) IVH.

A single ophthalmologist, DC, examined all cases and recorded the stage and location of ROP according to the International Classification of Retinopathy of Prematurity. ${ }^{12}{ }^{13}$ Patients with stage 3 threshold ROP were treated with cryotherapy between 1989 and 1992. Cryotherapy was applied over the non-vascularised retina. After 1992 patients with stage 3 threshold disease were treated with argon or diode laser. Laser was applied to the avascular retina anterior to the ridge in a scatter fashion.

\section{Statistics}

Statistical analysis of the data was performed using SPSS for Windows Version 11.0. For the purpose of this study the two eyes of the same patient have been used as independent variables. Pearson correlation coefficient $(r)$ was calculated to quantify the relation between grating acuity score at 6 months and optotype acuity at 36 months. For calculation of predictive value, negative tests were considered to be those resulting in acuity $<6 / 9$ at 36 months and positive tests were those with acuity $\geqslant 6 / 9$ at 36 months. Predictive value was calculated by dividing the number of eyes with $\mathrm{VA} \geqslant 2 \mathrm{c} / \mathrm{d}$ at 6 months and VA $\geqslant 6 / 9$ at 36 months by the number of eyes with $\geqslant 2 \mathrm{c} / \mathrm{d}$ at 6 months only. Binary logistic regression was used to compare the frequency of high myopia in the treated and untreated groups. $p$ Values of $\leqslant 0.05$ were taken to be significant.

\section{RESULTS}

In all, 139 babies born between 1989 and 1999 had stage 3 ROP. Six children died and 15 had a poor outcome despite treatment and progressed to stage 5. These eyes were excluded, as the aim of this study was to report the two regressed groups with and without treatment. Thirty three infants (three cryotherapy group, four laser group, and 26 in the subthreshold group) were either lost to follow up or had incomplete records and were excluded. Follow up data on treated infants were greater as there was greater compliance in clinic attendance. Thus, 3 year follow up data were available for 85 infants. Five infants had stage 3 threshold ROP in one eye and stage 3 subthreshold ROP in their fellow eye and were included in their respective group. Table 1 contains a summary of the demographic data.

\section{Cryotherapy group}

Forty eyes with stage 3 threshold ROP and treated with cryotherapy were included in the study. This included both eyes of 17 infants (34 eyes). Six patients had asymmetric disease and the treated threshold eyes (six eyes) were included. The fellow eye had stage 2 ROP in one, subthreshold stage 3 ROP in three and in three patients progressed to stage 5 despite treatment.

\section{Laser group}

Eighty one eyes with stage 3 threshold ROP were treated with either diode or argon laser; 38 infants (76 eyes) had bilateral stage 3 ROP and five infants had asymmetric ROP; the eye with treated threshold disease was included. The fellow eye had stable subthreshold stage 3 ROP in three and stage 2 ROP in two .

\section{Subthreshold stage 3 ROP group}

A total of 34 eyes had spontaneously regressed subthreshold stage 3 ROP. It was bilateral in 10 infants (20 eyes) and unilateral in 14 infants (14 eyes). This included the five infants who had treated threshold disease in their fellow eye.

\section{Visual acuity (fig 1 A, B)}

Grating visual acuity data at 6 months and Snellen acuity data at 36 months were available for 155 eyes (81 laser; 40 cryotherapy; 34 subthreshold). A VA $\geqslant 2 \mathrm{c} / \mathrm{d}$ at 6 months was expected to predict a VA of $\geqslant 6 / 9$ at 36 months and was considered to be a favourable outcome. Table 2 presents the Pearson correlation coefficients between 6 month and 36 month VA estimates. Correlation coefficients relating grating acuity scores and Kay picture test acuity were significant for all groups.

In the babies who underwent cryotherapy $12 / 40$ eyes (30\%) achieved a grating acuity score of $2 \mathrm{c} / \mathrm{d}$ or better at 6 months. At 36 months, $9 / 40$ (22.5\%) had VA equal to or better than 6/9 Snellen equivalent. After laser treatment 47 (58\%) of the 81 eyes had a grating acuity score $2 \mathrm{c} / \mathrm{d}$ or better at 6 months;

Table 1 Subject demographic characteristics

\begin{tabular}{llll}
\hline & \multicolumn{2}{l}{ Stage 3 threshold ROP } & \\
\cline { 2 - 3 } & Laser & Cryo & Subthreshold stage 3 ROP \\
\hline Infants & 43 & 23 & 24 \\
Eyes & 81 & 40 & 34 \\
Mean (SD) birth weight (g) & $862(204)$ & $790(200)$ & $929(294)$ \\
Mean (SD) gestational age (weeks) & $26(1.8)$ & $25(1.3)$ & $26(2.5)$ \\
\hline
\end{tabular}



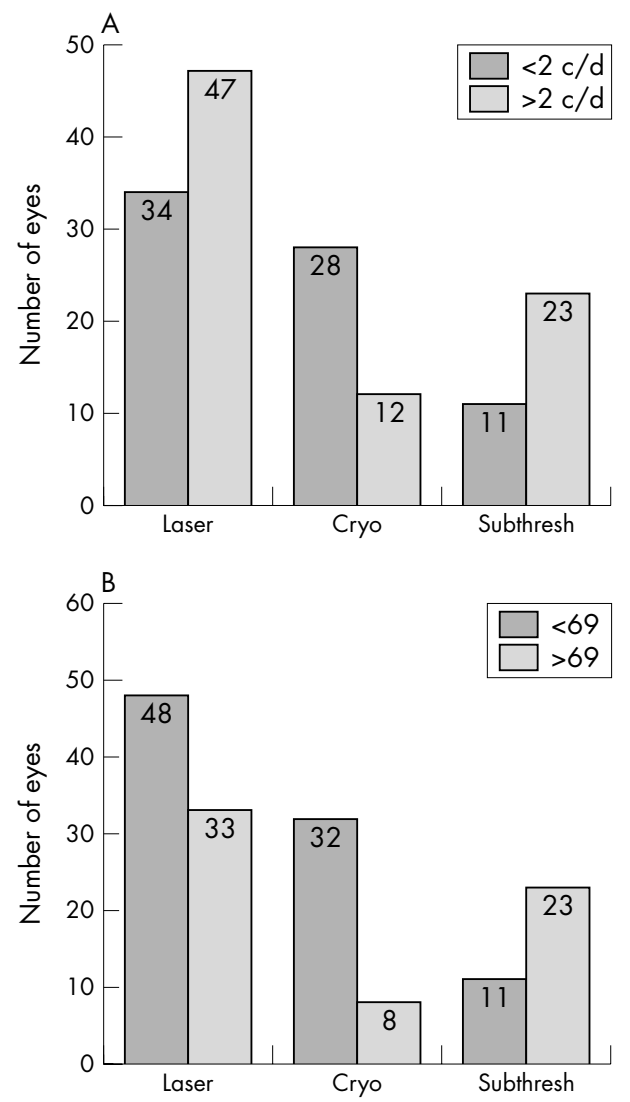

Figure 1 Distribution of visual acuity for eyes with treated threshold stage 3 ROP (laser 81 eyes; cryotherapy 40 eyes), and eyes with subthreshold stage 3 ROP (34 eyes). (A) At 6 months grating acuity scores $\geqslant 2$ cycles/degree were taken to be favourable. (B) At 36 months optotype acuity of $\geqslant 6 / 9$ were categorised as favourable.

$33 / 81(40.07 \%)$ achieved $6 / 9$ or better at 3 years. Babies with spontaneously regressed subthreshold stage 3 ROP had better VA than the treated group; 22 out of 34 eyes $(64.7 \%)$ achieved $>2 \mathrm{c} / \mathrm{d}$ at 6 months and 23/34 (67.6\%) eyes were 6/9 or better at 36 months. As shown in table 2, the positive and negative predictive values were variable. Overall, in $84 \%$ eyes the grating acuity scores at 6 months had a predictive value for the optotype acuity at 36 months. Predictive values for negative tests were higher for the treated groups and predictive value for positive test was higher for the untreated subthreshold group. Eyes with subthreshold stage 3 ROP were 2.2 times more likely to have $6 / 9$ or better than the treated eyes. There was a significant difference in the odds of achieving a VA of $6 / 9$ at 36 months when infants with regressed threshold stage 3 ROP following treatment were compared to infants who had spontaneously regressed subthreshold stage 3 ROP (OR 3.9 (95\% CI: 1.75 to $8.84))$.

\section{Refractive error}

There was a statistically significant difference in the refractive error between laser treatment and cryotherapy at 6 months $(p=0.023)$ and 36 months $(p=0.005)$ (table 3$)$. Patients who had subthreshold stage 3 not requiring treatment were significantly less myopic than the treated groups (that is, laser and cryotherapy) with a mean spherical equivalent refractive error of +0.71 at 6 months $(p=0.007)$ and -0.22 at 36 months $(\mathrm{p}=0.019)$ (fig 2 ).

Eighteen eyes out of 155 eyes ( $11.9 \%$ ) had high myopia at 6 months compared to $47 / 155$ eyes $(28.7 \%)$ at 36 months. The difference in the refractive error at 6 months and 36 months was statistically significant $(p<0.005)$. At 36 months the proportion of eyes with high myopia $(\geqslant-5 \mathrm{D})$ was $52.5 \%(21 / 40)$ in the cryotherapy treated eyes, $29.6 \%(24 / 81)$ in the laser treated eyes and $(2 / 34=5.8 \%)$ in the eyes with spontaneously regressed subthreshold stage 3 ROP. When comparing the treated eyes (laser and cryotherapy) with the subthreshold eyes there was a significantly higher incidence of high myopia $(>-5 D)$ in the treated group $(\mathrm{p}=0.003)$.

\section{Strabismus}

Twenty five out of the 85 infants had strabismus at 6 months and $42 / 85$ patients had strabismus at 36 months. Twenty three infants had convergent squint and two had divergent squint at 6 months.

There was a statistically significant association between strabismus and anisometropia at 36 months ( $>3 \mathrm{D})$ $(\mathrm{p}=0.016)$.

Cranial ultrasound results were available for 71 children; $18(25.35 \%)$ had no IVH, $30(42.25 \%)$ had mild to moderate IVH (I or II), and $23(32.39 \%)$ had grades III-IV intraventricular haemorrhage.

Seventy six per cent (19/25) of infants with strabismus at 6 months had had IVH at birth. IVH was grade III-IV in 10 of the 25 infants $(40 \%)$. This included all patients with subthreshold stage 3 ROP and squint. At 36 months, 19 $(82.6 \%)$ of 23 infants with grade III-IV IVH at birth had strabismus compared with 19 (39.5\%) of 48 with mild or moderate IVH. This higher incidence of strabismus in high grade IVH was statistically significant $(p=0.01)$.

\section{Anatomical outcome}

Fourteen eyes had a dragged macula on funduscopy: six (7.4\%) eyes in the laser group and three $(20 \%)$ eyes in the cryotherapy group. None of the eyes with subthreshold stage 3 ROP had macular scarring or ectopia. The incidence of macular ectopia was 2.7 times higher in the group treated with cryotherapy compared to eyes treated with laser.

\section{DISCUSSION}

Myopia, ${ }^{14-16}$ visual impairment, ${ }^{4}$ strabismus, ${ }^{17}$ and $\mathrm{IVH}^{18}$ are recognised complications in premature low birthweight infants. In the multicentre, Cryo-ROP Study, cryotherapy was shown to benefit patients with threshold ROP. ${ }^{12}$ In recent years, laser photocoagulation has become increasingly

Table 2 Pearson correlation coefficients between 6 month grating acuity scores and 36 month Kay picture test VA estimates

\begin{tabular}{llllll}
\hline & No & $\boldsymbol{r}$ & p Value & PPV & NPV \\
\hline All eyes & 155 & 0.6 & $<0.005$ & 0.69 & 0.88 \\
Cryo Rx & 81 & 0.5 & $<0.005$ & 0.64 & 0.91 \\
Laser Rx & 40 & 0.6 & $<0.005$ & 0.67 & 0.96 \\
Subthreshold group & 34 & 0.6 & $<0.005$ & 0.82 & 0.58 \\
\hline PPV, positive predictive value of VA $\geqslant 2$ c/d for predicting a VA $\geqslant 6 / 9$ at 36 months. NPV, negative predictive \\
value for VA <2 c/d for predicting a VA <6/9.
\end{tabular}


Table 3 Spherical equivalent (SE) refractive error at 6 months and 36 months

\begin{tabular}{|c|c|c|c|c|c|c|}
\hline & \multicolumn{3}{|l|}{6 months } & \multicolumn{3}{|l|}{36 months } \\
\hline & $\begin{array}{l}\text { Cryo } \\
(n=40)\end{array}$ & $\begin{array}{l}\text { Laser } \\
(\mathrm{n}=81)\end{array}$ & $\begin{array}{l}\text { Subthreshold } \\
(n=33)\end{array}$ & $\begin{array}{l}\text { Cryo } \\
(n=40)\end{array}$ & $\begin{array}{l}\text { Laser } \\
\text { ( } \mathrm{n}=81)\end{array}$ & $\begin{array}{l}\text { Subthreshold } \\
(\mathrm{n}=33)\end{array}$ \\
\hline $\begin{array}{l}\text { Refraction } \\
\text { (mean SE (D)) }\end{array}$ & $\begin{array}{l}-3.25 \\
(-13 \text { to }+3.8)\end{array}$ & $\begin{array}{l}-0.91 \\
(-10.75 \text { to }+4)\end{array}$ & $\begin{array}{l}+0.71 \\
(-6 \text { to }+4)\end{array}$ & $\begin{array}{l}-6.5 \\
(-21.5 \text { to } 1.4)\end{array}$ & $\begin{array}{l}-2.43 \\
(-13 \text { to }+4)\end{array}$ & $\begin{array}{l}-0.22 \\
(-9 \text { to }+2.25)\end{array}$ \\
\hline
\end{tabular}

popular for threshold ROP because of its ease of delivery, less refractive error and better functional outcome as compared to cryotherapy. ${ }^{519-21}$

Our study compares the visual outcome, the prevalence of myopia and strabismus at two ages, 6 months and 3 years, in children with regressed stage 3 ROP subthreshold versus threshold disease.

\section{Vision}

$22.5 \%$ in the cryotherapy group, $40 \%$ in the laser group, and $68 \%$ in the subthreshold group achieved a favourable visual outcome at 36 months. In our study we defined a favourable visual outcome at 3 years as best corrected VA $\geqslant 6 / 9$ (equivalent to 20/30). Other studies have not been as strict with their definitions of favourable outcome. Connolly et al 20 considered a good visual outcome as $V A \geqslant 20 / 50$ (equivalent to $6 / 15$ ) and found this in $50 \%$ eyes after laser photocoagulation. However, they do not specify the age of the infant at the final visual assessment which could also account for the increased proportion of infants who could pass the test. Cottrell et $a l^{22}$ had VA of $\geqslant 6 / 12$ in $45.5 \%$ in the cryotherapy group and $56.7 \%$ in the laser group. There were fewer infants and eyes in both groups in their study and once again they do
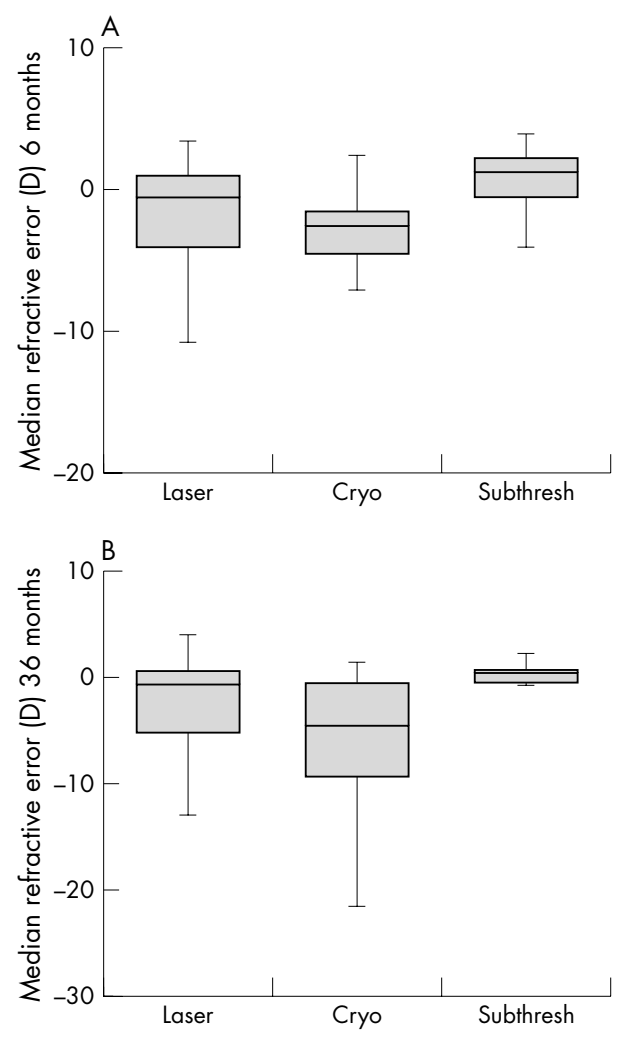

Figure 2 (A) Median refractive error and ranges at 6 months. (B) Median refractive error and ranges at 36 months. not specify the age of the infant at the final visual assessment. Our results following cryotherapy correlate more closely with the Cryo-ROP Study Group. ${ }^{1}$ We speculate that the better VA in the laser group could be because of advances in neonatal practice, less severe disease, timely referral of threshold disease from outside units, and methods of treatment.

Longitudinal data reported by Birch and Spencer, ${ }^{23}$ Zubcov et $a l_{,}{ }^{24}$ and Dobson et al ${ }^{25}$ suggest that early grating acuity tests may be predictive of long term functional outcome. In our study, long term reliability of grating acuity scores was tested by calculating correlation between VA $\geqslant 2 \mathrm{c} / \mathrm{d}$ at 6 months with VA at $\geqslant 6 / 9$ at 36 months. Our findings suggest that grating acuity scores may be valid predictors of optotype VA later in childhood. The predictive value for the positive test was high in the subthreshold group (0.82). The predictive value of a negative test was high especially for the treated infants, 0.91 in the cryotherapy treated eyes and 0.96 in the laser treated eyes. Overall, the grating acuity scores $<2 \mathrm{c} / \mathrm{d}$ at 6 months had a predictive value for the optotype acuity $<6 / 9$ at 36 months $(0.88)$. The variability in the predictive values between the treated groups and the untreated group could be because of the relatively low number of eyes that had VA $>6 / 9$ in the former and VA $<6 / 9$ in the later. Thus, few eyes were included in the calculation of their respective predictive values. Nevertheless, despite this variability, the predictive values of positive test and negative tests approximate $60 \%$, indicating that grating acuity scores are moderately predictive of 36 month optotype acuity.

The visual outcome depends not only on the regression of the retinopathy with normal posterior retinal anatomy but also on the neurological status of the infants. The latter has been observed by Luna et $a^{26}$ who reported a delay in VA development in patients with grade III or IV IVH. O'Keefe et al highlighted ${ }^{27}$ the association of all grades of IVH with optic atrophy and hydrocephalus.

\section{Myopia}

In all groups there was a myopic shift by 36 months. The prevalence of high myopia increased from $11 \%$ at 6 months to $28.7 \%$ at 36 months. This is consistent with previously published reports describing a trend of increasing myopia in both laser treated and cryotherapy treated eyes. ${ }^{11} 2829$ Schaffer et $a l^{30}$ found the degree and frequency of myopia to be similar in premature infants with low grade retinopathy of prematurity (ROP) and no retinopathy. In our study, only one eye in the spontaneously regressed subthreshold group developed high myopia. In our previous report, ${ }^{3}$ we noted that there was a statistically significant difference in the mean spherical refractive error between laser and cryotherapy at 3 months and 12 months. We found this trend continued when the two groups were compared at 36 months. Connolly et al have compared cryotherapy with laser treatment at 5 year $^{2}$ and 10 year $^{21}$ follow ups and found similar results.

The aim of this study was also to compare the refraction in patients with stage 3 ROP treated with laser or cryotherapy against those with subthreshold regressed disease at 6 months and 3 years post-term. We found that patients 
who had regressed subthreshold stage 3 not requiring treatment were significantly less myopic than the treated group at both ages.

Previous studies have indicated that axial length does not explain all the myopia found in ROP. ${ }^{31}{ }^{32}$ Kent et $a l^{33}$ and Connolly et $a l^{21}$ found that the crystalline lens power was greater in the cryotherapy treated eyes than laser treated eyes and in both groups the lens power was greater than normal. The tissue destruction associated with cryotherapy compared to laser, ${ }^{34}$ may also play a part in the higher incidence of myopia in this group. Quinn et $a l^{35}$ reported that there was almost an equal prevalence of myopia in the treated and control eyes in the Cryo-ROP study. In our study the relatively low incidence of myopia in the subthreshold group suggests that threshold stage 3 ROP irrespective of the method of treatment, may exert other influences on ocular growth such as anterior segment arrest which contributes to the development of high myopia

\section{Strabismus}

ROP, refractive error, low birth weight, anisometropia, and cerebral palsy have all been independently associated with the presence of strabismus. ${ }^{36}$ In this study, at 36 months the overall prevalence of strabismus was $50 \%$ in patients with stage 3 ROP (subthreshold and threshold). Our results were similar to $\mathrm{Ng}$ et $\mathrm{al}^{5}$ and White and Repka ${ }^{37}$ who had an incidence of $62 \%$ and $58 \%$ in their respective studies. We also found a statistically significant association between strabismus and anisometropia $(\mathrm{p}=0.016)$. In addition, we correlated the presence of strabismus with the presence of neurological signs of injury; $76 \%$ were associated with IVH or cerebral palsy or nystagmus. All patients with subthreshold stage 3 ROP and squint had some degree of IVH. Eighty three per cent of infants with severe IVH had strabismus compared with $39.5 \%$ with no or mild to moderate IVH. This higher incidence of strabismus in severe IVH was statistically significant $(p=0.01)$. This was unlike the results of O'Keefe et $a l^{27}$ who found no statistically significant difference in the incidence of strabismus between low and high grade IVH. Various other studies have demonstrated a strong correlation between the severity of the haemorrhages and the subsequent development of strabismus. ${ }^{36-40}$ Our study suggests that in patients with lower grades of retinopathy not approaching threshold, neurological injury may play a greater part in the development of strabismus.

\section{Anatomical outcome}

In this study, $7.4 \%$ of the laser treated eyes and $20 \%$ of the cryotherapy treated eyes had macular ectopia. This is consistent with previously published reports. ${ }^{7237}$ The cryotherapy treated eyes were 2.7 times more likely to develop macular ectopia as opposed to the laser treated eyes. None of the patients with prethreshold stage 3 ROP developed macular dragging.

Some recent studies have suggested prophylactic treatment for subthreshold ROP. ${ }^{694142}$ This could result in treating some eyes that would have otherwise undergone spontaneous regression. The ETROP study has demonstrated a better functional and structural outcome at 9 months of age. Our study is not directly comparable with ETROP, although there may be some overlap within the groups. We await with interest their long term visual results.

In summary, our study confirms that laser treatment for threshold ROP has a better structural and functional outcome compared to cryotherapy. Furthermore eyes with spontaneously regressed subthreshold stage 3 ROP have a good functional and structural outcome. This study also found that grating acuity scores within the normal range at 6 months could indicate a normal acuity at 3 years of age and highlights the association between all grades of IVH (especially severe IVH) and strabismus in infants with ROP.

\section{ACKNOWLEDGEMENTS}

The authors thank the orthoptic and optometry staff at Walton Hospital for their invaluable assistance. They also thank Mr Brian Fleck and Ms Cathy Williams, for their helpful comments on the previous versions of this article.

\section{Authors' affiliations}

J Sahni, N V Subhedar, D Clark, Department of Ophthalmology,

Walton Hospital, Rice Lane, Liverpool L9 IAE, UK

\section{REFERENCES}

1 Cryotherapy for Retinopathy of Prematurity Cooperative Group. Multicentre trial of cryotherapy for retinopathy of prematurity. 3.5 year outcomestructure and function. Arch Ophthalmol 1993;111:339-44.

2 Connolly BP, McNamara JA, Sharma S, et al. A comparison of laser photocoagulation with trans-scleral cryotherapy in the treatment of threshold retinopathy of prematurity. Ophthalmology 1998;105:1628-31.

3 Laws F, Laws D, Clark D. Cryotherapy and laser treatment for acute retinopathy of prematurity: refractive outcomes, a longitudinal study. Br J Ophthalmol 1997;81:12-15.

4 Pearce IA, Pennie FC, Gannon LM, et al. Three year visual outcome for stage 3 retinopathy of prematurity; cryotherapy versus laser. $\mathrm{Br} J$ Ophthalmol 1998:82:1254-9.

$5 \mathrm{Ng} \mathrm{EY}$, Connolly BP, McNamara JA, et al. A comparison of laser photocoagulation with cryotherapy for threshold retinopathy of prematurity at 10 years: part 1. Visual function and structural outcome. Ophthalmology 2002; 109:831-3.

6 Early Treatment of Retinopathy of Prematurity Cooperative Group. Revised indications for the treatment of retinopathy of prematurity. Arch Ophthalmol 2003; 121:1684-96.

7 Rescan Z, Vamos R, Salacz G. Laser treatment of zone I prethreshold and stage 3 threshold retinopathy of prematurity. J Paediatr Ophthalmol Strabismus 2003;40:204-7.

8 Vander JF, Handa J, McNamara JA, et al. Early treatment of posterior retinopathy of prematurity: a controlled trial. Ophthalmology 1997; 104:1731-6.

9 Gurdian SJ, Gehlbach PL, Hunyor AP, et al. Retinal Detachment associated with subthreshold retinopathy of prematurity. J Pediatr Ophthalmol Strabismus 2001;38:279-83.

10 Vistech Consultants. Teller acuity card handbook. Dayton, $\mathrm{OH}$ : Vistech Consultants, 1989.

11 Graham E Quinn, Dobson V, Kilvin J, et al. Prevalence of myopia between 3 months and $51 / 2$ years in preterm infants with and without retinopathy of prematurity. Ophthalmology 1998;105:1292-300.

12 International Committee. An international classification of retinopathy of prematurity. Br J Ophthalmol 1984;68:690-7.

13 The International Committee for the Classification of the Late Stages of Retinopathy of Prematurity. An International Classification of Retinopathy of Prematurity. II. The classification of retinal detachment. Arch Ophthalmo 1987; 105:906-12.

14 Gordon RA, Donzis PB. Myopia associated with retinopathy of prematurity. Ophthalmology 1986;93:1593-8.

15 Koraszewska MB, Samochowiec DE, Pieczara E, et al. Myopia as a complication of retinopathy of prematurity. Klin Oczna 1993;95:339-42.

16 Nissenkorn I, Yassur Y, Mashkowski D, et al. Myopia in premature babies with and without retinopathy of prematurity. $\mathrm{Br} J$ Ophthalmol 1983;67:170-3.

17 O'Connor AR, Stephenson TJ, Johnson A, et al. Strabismus in children of birth weight less than 1701g. Arch Ophthalmol 2002;120:767-73.

18 Christiansen SP, Fray KJ, Spencer T. Ocular outcomes in low birth weight premature infants with intraventricular haemorrhage. J Pediatr Ophthalmol Strabismus 2002;39:157-65.

19 Hammer ME, Pusateri TJ, Hess JB, et al. Threshold retinopathy of prematurity. Transition from cryopexy to laser treatment. Retina 1995; 15:486-9.

20 Connolly BP, McNamara, Regillo CD, et al. Visual outcomes after laser photocoagulation for threshold retinopathy of prematurity. Ophthalmology 1999; 106:1734-8.

21 Connolly BP, Eugene YJN, MCNamara JA, et al. A comparison of laser photocoagulation with cryotherapy in the treatment of threshold retinopathy of prematurity at 10 years. Part 2. Refractive outcome. Ophthalmology 2002;109:936-41.

22 Gnanaraj L, Brennan R, Cottrell DG. Retinopathy of prematurity in practice. II: long-term results following treatment for threshold disease. Eye 2003; 17:189-93.

23 Birch EE, Spencer R. Visual outcome in infants with cicatricial retinopathy of prematurity. Invest Ophthalmol Vis Sci 1991;32:410-5.

24 Zubcov AA, Rossillion BM, Kacer B, et al. Predictive value of Teller Acuity Card Test (TACT) and comparison of recognition and grating acuities in premature children with and without residua of retinopathy of prematurity (ROP). Klin Monatsbl Augenheilkd 2002;219:722-7. 
25 Dobson V, Quinn GE, Siatkowski M, et al. Agreement between grating acuity at age 1 year and Snellen acuity at age 5.5 years in the preterm child. Invest Ophthalmol Vis Sci 1999;40:496-503.

26 Luna B, Dobson V, Biglan AW. Development of grating acuity in infants with regressed stage 3 retinopathy of prematurity. Invest Ophthalmol Vis Sci 1990:31:2082-7.

27 Michael O'Keefe, Namir Kafil-Hussain, et al. Ocular significance of intraventricular haemorrhage in premature infants. $\mathrm{Br} J$ Ophthalmol 2001;85:357-9

28 Knight-Nanan DM , $\mathrm{O}^{\prime}$ Keefe $M$. Refractive outcome in eyes with retinopathy of prematurity treated with cryotherapy or diode laser: 3 year follow up. Br J Ophthalmol 1996;80:998-1001.

29 Choi MY, Park IK, Yu YS. Long term refractive outcome in eyes of preterm infants with and without retinopathy of prematurity: comparison of keratometric value, axial length, anterior chamber depth, and lens thickness. Br J Ophthalmol 2000;84:138-43.

30 Schaffer DB, Quinn GE, Johnson L. Sequelae of arrested mild retinopathy of prematurity. Arch Ophthalmol 1984;102:373-6.

31 Hittner MH, Rhodes LM, McPherson AR. Anterior segment abnormalities in cicatricial retinopathy of prematurity. Ophthalmology 1979:86:803-16

32 Kelly SP, Fielder AR. Microcornea associated with retinopathy of prematurity Br J Ophthalmol 1987;71:201-3.

33 Kent $D$, Pennie $F$, Laws $D$, et al. The influence of retinopathy of prematurity on ocular growth. Eye 2000;14:23-9.
34 Brancato R, Praseti R, Leoni G, et al. Histopathology of diode and argon laser lesions in rabbit retina. A comparative study. Invest Ophthalmol Vis Sci 1989;30:1504-10.

35 Quinn GE, Dobson V, et al. Cryotherapy for Retinopathy of Prematurity Cooperative Group. Does cryotherapy affect refractive error? Results from treated versus control eyes in the cryotherapy for retinopathy of prematurity treated versus control eyes in the cryothero
trial. Ophthalmology 2001;108:343-9.

36 O'Connor AR, Stephenson TJ, et al. Strabismus in children of birth weight less than $1701 \mathrm{~g}$. Arch Ophthalmol 2002;120:767-73

37 White JE, Repka MX. Randomised comparison of diode laser photocoagulation versus cryotherapy for threshold retinopathy of prematurity: 3-year outcome. J Pediatr Ophthalmol Strabismus 1997;34:75.

38 Phillips J, Christiansen SP, Ware G, et al. Ocular morbidity in very low birth weight infants with intraventricular haemorrhage. Am J Ophthalmol 1997; 123:218-33.

39 Page JM, Schneeweiss S, Whyte HE, et al. Ocular sequelae in premature infants. Pediatrics 1993;92:787-90

40 Tamura EE, Hoyt CS. Oculomotor consequences of intraventricular haemorrhages in premature infants. Arch Ophthalmol 1987;105:533-5.

41 Tasman W. Threshold retinopathy of prematurity revisited. Arch Ophthalmol 1992;110:623-4.

42 Stenberg P, Lopez PF, Lambert PF, et al. Controversies in the management of retinopathy of prematurity. Am J Ophthalmol $1992 ; 113: 589-92$

\section{Call for papers}

10th European Forum on Quality Improvement in Health Care 13-15 April 2005, ExCel, Docklands, London For further information on how to submit your paper please go to: http://www.quality.bmipg.com 